\title{
The maize W22 genome provides a foundation for functional genomics and transposon biology
}

\author{
Nathan M. Springer', Sarah N. Anderson ${ }^{1}{ }^{1}$, Carson M. Andorf², Kevin R. Ahern ${ }^{3}{ }^{3}$, Fang Bai ${ }^{4}$, \\ Omer Barad5, W. Brad Barbazuk ${ }^{6}$, Hank W. Bass ${ }^{7}$, Kobi Baruch5, Gil Ben-Zvi ${ }^{5}$, Edward S. Buckler (1) ${ }^{8,9}$, \\ Robert Bukowski ${ }^{9}$, Michael S. Campbell ${ }^{10}$, Ethalinda K. S. Cannon ${ }^{1}{ }^{2}$, Paul Chomet ${ }^{5}$, R. Kelly Dawe ${ }^{11}$, \\ Ruth Davenport ${ }^{6}$, Hugo K. Dooner ${ }^{12,13}$, Limei He Du ${ }^{12,13}$, Chunguang Du ${ }^{14}$, Katherine A. Easterling ${ }^{7}$, \\ Christine Gault ${ }^{6}$, Jiahn-Chou Guan ${ }^{4}$, Charles T. Hunter ${ }^{15}$, Georg Jander ${ }^{3}{ }^{3}$, Yinping Jiao ${ }^{10}$, Karen E. Koch ${ }^{4}$, \\ Guy Kol${ }^{5}$, Tobias G. Köllner (16) ${ }^{16}$, Toru Kudo ${ }^{4,17}$, Qing Li' ${ }^{1}$, Fei Lu ${ }^{9,18,19}$, Dustin Mayfield-Jones ${ }^{20}$, \\ Wenbin Mei ${ }^{6}$, Donald R. McCarty ${ }^{4}{ }^{4}$, Jaclyn M. Noshay', John L. Portwood II2, Gil Ronen ${ }^{5}$, \\ A. Mark Settles ${ }^{4}{ }^{4}$, Doron Shem-Tov ${ }^{5}$, Jinghua Shi2 ${ }^{21}$, llya Soifer ${ }^{5}$, Joshua C. Stein ${ }^{10}$, Michelle C. Stitzer ${ }^{22}$, \\ Masaharu Suzuki ${ }^{4}$, Daniel L. Vera ${ }^{23}{ }^{23}$, Erik Vollbrecht ${ }^{24}$, Julia T. Vrebalov ${ }^{3}$, Doreen Ware ${ }^{8,10,25}$, \\ Sharon Wei ${ }^{10}$, Kokulapalan Wimalanathan $\mathbb{1}^{24}$, Margaret R. Woodhouse ${ }^{2}$, Wenwei Xiong ${ }^{14}$ \\ and Thomas P. Brutnell ${ }^{20,26 \star}$
}

The maize W22 inbred has served as a platform for maize genetics since the mid twentieth century. To streamline maize genome analyses, we have sequenced and de novo assembled a W22 reference genome using short-read sequencing technologies. We show that significant structural heterogeneity exists in comparison to the B73 reference genome at multiple scales, from transposon composition and copy number variation to single-nucleotide polymorphisms. The generation of this reference genome enables accurate placement of thousands of Mutator $(\mathbf{M u})$ and Dissociation $(\mathrm{Ds})$ transposable element insertions for reverse and forward genetics studies. Annotation of the genome has been achieved using RNA-seq analysis, differential nuclease sensitivity profiling and bisulfite sequencing to map open reading frames, open chromatin sites and DNA methylation profiles, respectively. Collectively, the resources developed here integrate W22 as a community reference genome for functional genomics and provide a foundation for the maize pan-genome.

\section{T} he maize W22 genome has served as a foundation for maize genetics since its early adoption by Brink for studies of paramutation $^{1}$. The inbred, developed at the Wisconsin Agricultural Experiment Station, lacked the ubiquitous suppressors of anthocyanin pigmentation present in most standard corn belt inbreds but did carry mutations in two regulatory genes controlling anthocyanin production, $R 1$ and $C 1$. Thus, Brink introgressed functional alleles of $R 1$ and $C 1$ to produce a color-converted W22 inbred (PI accession 674445). This inbred was soon adopted by Nelson, Kermicle and Dooner and their students, who led foundational studies to understand the genetics of high-protein maize $(\mathrm{O} 2)^{2}$, provide the first example of imprinting ${ }^{3}$, conduct the first transposon tagging experiments in plants ${ }^{4}$ and understand the mechanisms of Activator and Dissociation ${ }^{5,6}$ transposition. Today, the W22 genome is the host to thousands of mobilized, indexed Mutator ${ }^{7-9}$, Activator $^{10,11}$ and Dissociation ${ }^{12,13}$ insertions that are used extensively for functional genomics studies. Maize exhibits exceptional levels of structural variation that influences gene content and gene regulation among

\footnotetext{
'Department of Plant and Microbial Biology, University of Minnesota, Saint Paul, MN, USA. ${ }^{2}$ USDA-ARS, Corn Insects and Crop Genetics Research Unit and lowa State University, Department of Computer Science, lowa State University, Ames, IA, USA. ${ }^{3}$ Boyce Thompson Institute, Ithaca, NY, USA. ${ }^{4}$ Horticultural Sciences Department, University of Florida, Gainesville, FL, USA. ${ }^{5}$ NRGene Ltd, Ness Ziona, Israel. ${ }^{6}$ Department of Biology and the UF Genetics Institute, University of Florida, Cancer \& Genetics Research Complex, Gainesville, FL, USA. ${ }^{7}$ Department of Biological Science, The Florida State University, Tallahassee, FL, USA. ${ }^{8}$ USDA-ARS, Holley Center for Agriculture and Health, Ithaca, NY, USA. ${ }^{9}$ Institute for Genomic Diversity, Biotechnology Building, Cornell University, Ithaca, NY, USA. ${ }^{\circ}$ Cold Spring Harbor Laboratory, Cold Springs Harbor, NY, USA. "Department of Plant Biology, University of Georgia, Athens, GA, USA. ${ }^{12}$ Department of Plant Biology, Rutgers University, New Brunswick, NJ, USA. ${ }^{13}$ Waksman Institute, Rutgers University, Piscataway, NJ, USA. ${ }^{14}$ Department of Biology, Montclair State University, Montclair, NJ, USA. ${ }^{15}$ USDA-ARS Chemistry Research Unit, Gainesville, FL, USA. ${ }^{16}$ Department of Biochemistry, Max Planck Institute for Chemical Ecology, Jena, Germany. ${ }^{17}$ Metabologenomics, Inc., Tsuruoka, Yamagata, Japan. ${ }^{18} \mathrm{CAS}-\mathrm{JIC}$ Centre of Excellence for Plant and Microbial Science (CEPAMS), Shanghai Institutes for Biological Sciences, Chinese Academy of Sciences, Shanghai, China. ${ }^{19}$ The State Key Laboratory of Plant Cell and Chromosome Engineering, Institute of Genetics and Developmental Biology, Chinese Academy of Sciences, Beijing, China. ${ }^{20}$ Donald Danforth Plant Science Center, St. Louis, MO, USA. ${ }^{21}$ Bionano Genomics, San Diego, CA, USA. ${ }^{22}$ Department of Plant Sciences and Center for Population Biology, University of California, Davis, Davis, California, USA. ${ }^{23}$ Center for Genomics and Personalized Medicine, The Florida State University, Tallahassee, FL, USA. ${ }^{24}$ Department of Genetics, Development and Cell Biology, lowa State University, Ames, IA, USA. ${ }^{25}$ USDA-ARS, NEA Robert W. Holley Center for Agriculture and Health, Cornell University, Ithaca, NY, USA. ${ }^{26}$ Present address: College of Agronomic Sciences State Key Laboratory of Crop Biology, Shandong Agricultural University, Shandong, China. *e-mail: brutnell@gmail.com
} 
different inbred lines ${ }^{14-17}$. This limits the utility of the B73 reference genome $^{18}$ or the $\mathrm{PH} 207$ genome $^{19}$ for interpreting data derived for mutant alleles in a W22 genetic background. Detailed knowledge of the W22 genome structure and content is critical to fully exploit reverse genetics resources and biological processes in W22.

\section{W22 genome de novo assembly and validation}

The W22 genome was sequenced and assembled using deep sequencing $(>180 \times)$ of Illumina short-read sequences from libraries with a variety of insert sizes using the assembly algorithms developed by NRGene ${ }^{19}$ (see Methods and Reporting Summary for details). Sequence contigs were developed based on paired-end (PE) short reads and then were put into scaffolds using PE or matepair (MP) links. Further ordering and orientation of scaffolds was achieved through alignment to the B73 genome assembly. The initial assembly was improved through utilization of the $10 \times$ genomics library platform ${ }^{20}$ and sequencing using the HiSeq X10 to bridge contigs and correct initial misassemblies (see Methods). This revealed 34 potential chimeric scaffolds that were broken and reassembled to generate a W22v2 genome (Table 1; see Methods). The W22 whole genome is available at NCBI under the accession GCA_001644905.2. The genome and accompanying resources are available at MaizeGDB under the name Zm-W22-REFERENCENRGENE-2.0 with the identifier Zm00004b.

Several approaches were used to assess the quality and completeness of the W22 genome sequence at macro and micro scales. To confirm the overall quality of the sequence assembly, we created an independent optical map based on the restriction site BspQI ${ }^{21}$ and aligned the optical map with an in silico digested version of the genome assembly. The two assemblies strongly supported each other, showing exceptional (>98\%) alignment and colinearity. Further confirmation of the assembly was performed using a suite of 4.4 million SNP markers validated against the maize nested association mapping panel ${ }^{22}$. A genetic assessment of contig and scaffold ordering was conducted by aligning 4.4 million SNP markers to an initial W22 assembly scaffold revealing $\sim 30$ inconsistencies (see Methods). This genetic map was used to correct the initial assembly to produce a W22v2 assembly that integrates the genetic and physical maps and provided evidence for robust assembly. The W22v2 genome has a larger number of gaps (as defined by $>10 \mathrm{Ns}$ ) than the B73v4 genome but substantially fewer gaps than the PH207 genome (Supplementary Table 1).

A more granular assessment of the assembly quality and accuracy was made by comparing the W22v2 assembly to a 238-kilobase $(\mathrm{kb})$ interval encompassing the W22-bz1 locus on chromosome 9 that had previously been sequenced using Sanger sequencing technology $y^{23}$. The vast majority $(\sim 113 \mathrm{Mb})$ of this region aligned with $>99 \%$ identity in a collinear fashion (Fig. 1). The primary difference between the W22v2 genome assembly and the earlier Sanger $\mathrm{BAC}$ assembly is an unfilled gap present in the W22v2 assembly that corresponds to 7,137 base pairs (bp) of a Misfit retrotransposon that is located in the midst of an $85-\mathrm{kb}$ retrotransposon cluster (Fig. 1). There are three other small gaps in this region that occur within repetitive elements but the size of these gaps is similar to the BAC sequence and the order and orientation is the same as the BAC. This analysis provides further evidence for the quality of the W22v2 assembly and suggests that remaining gaps likely occur within repetitive regions.

\section{Annotation of genes and transposons in W22}

Genes of the W22v2 genome were annotated using a modified Maker P approach that was developed for B73v4 ${ }^{18}$ together with RNA-seq from W22 tissues and full-length cDNA data (see Methods for details). A 'working gene set' of 57,181 genes was identified. Removal of putative transposons, low-confidence singleexon genes and potential pseudogenes resulted in a 'filtered gene set'
Table 1 | Summary statistics for the W22v2 assembly

\begin{tabular}{ll} 
Total scaffolds & 306 \\
Assembly size (bp) & $2,133,868,728$ \\
Size of gaps (bp) & $40,626,859$ \\
Size of gaps (\%) & 1.903 \\
Contig N50 (bp) & 72,426 \\
Scaffold N50 (bp) & $35,520,102$ \\
N50 (number of sequences) & 18 \\
Scaffold N90 (bp) & $10,997,073$ \\
\hline
\end{tabular}

a Gaps defined as $>10$ Ns.

of 40,789 genes. The exons of the filtered gene set account for $2.99 \%$ and the introns account for another $5.15 \%$ of the $2.2 \mathrm{~Gb}$ W22v2 assembly. The number of genes and distribution along the chromosomes (Fig. 2) are very similar to previous maize genome assemblies for $\mathrm{B}^{2} 3^{4}$ and $\mathrm{PH} 207^{19}$. The quality and completeness of the W22 genome was assessed through a BUSCO analysis ${ }^{24}$. Over $90 \%$ of BUSCO genes are represented by a complete single copy in the W22 genome and only $3 \%$ of BUSCO genes are missing in the W22v2 assembly (Supplementary Fig. 1). These rates are equivalent to the B73v4 genome (Supplementary Fig. 1), suggesting similar qualities of annotation completeness and quality in these genomes.

The availability of a de novo assembly for the W22 genome allows for comparisons of gene copy number, structure and splicing between W22 and B73. Previous work has found evidence for frequent copy number variation and presence/absence variation among maize inbreds $\mathrm{s}^{17,19,25}$. The B73 and W22 genomes were compared to each other and to the Sorghum BTx623 genome ${ }^{26}$ to document the presence or absence of syntenic orthologs among these three genomes (Supplementary Table 2). Over $60 \%$ of the genes are present at syntenic locations in both maize lines and Sorghum. Another $15-20 \%$ of genes are found in both B73 and W22 but are absent from the BTx623 assembly (Supplementary Table 2). The remaining genes are present in one maize line and absent in the other. The majority of these genes are present only in B73 $(6,440)$ or W22 $(8,372)$ and not found in the other maize inbred or in Sorghum. There are relatively fewer examples of genes that are present in Sorghum and only one maize inbred (B73, 881; W22, 1,412). The presence of locally duplicated genes was evaluated in both the W22 and B73 genome, for a range of intervening genes from zero (tandem duplicates) to twenty (Supplementary Tables 3 and 4). A subset (678) of the examples of tandem duplications are observed in both B73 and W22. However, there are many other cases of tandem duplication that are observed only in B73 $(1,394)$ or in W22 $(1,261)$. The tandem duplicates were classified according to their relative arrangement (Supplementary Table 5). Tandem duplicates in the same orientation (head to tail) are more common than genes in a convergent (tail-to-tail) or divergent (head-to-head) arrangement (Supplementary Table 5).

The presence of a tandem duplication in one genome and not in the other could reflect mechanisms to rapidly expand or contract gene copy variants and may serve as a foundation for functional diversification; for example, the terpene synthase family has variable tandem duplications that may affect function (see Supplementary Note for details).

RNA-seq data generated from several W22 tissues were used to document examples of alternative splicing in W22 and compare these alternative splicing events to those observed in $\mathrm{B} 73^{27}$. We identified 13,591 W22 genes with alternative splicing, representing $49.4 \%$ of expressed multi-exon genes. The types, and frequencies, of alternative splicing events noted in W22 (Supplementary Table 6) are similar to those noted for $\mathrm{B} 73^{27}$, albeit at lower numbers, which 


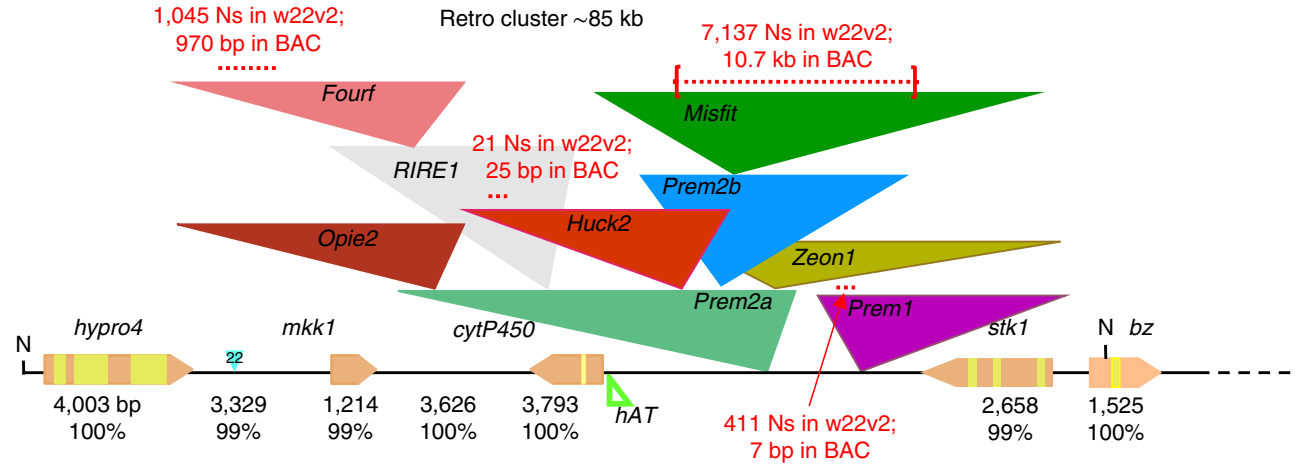

Fig. 1 | Benchmarking the W22v2 assembly quality and completeness. A comparison of the W22v2 assembly with a Sanger-based assembly of a 238-kb maize BAC ${ }^{23}$ (Genbank; EU338354). This region contains five genes (yellow/tan symbols along line) as well as a number of transposons (colored triangles). The W22v2 assembly matches the majority of the sequence at quite high identity (>99\%) and the locations of gaps in the W22v2 genome (indicated by red dotted lines).
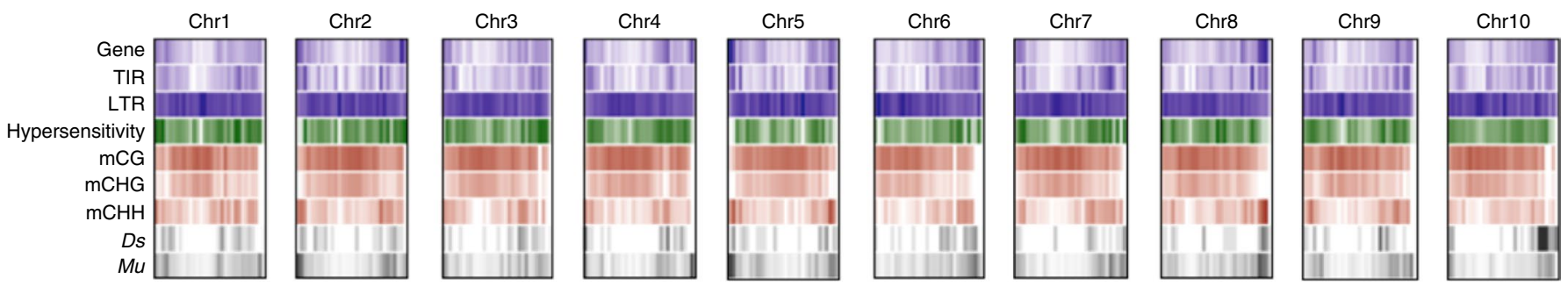

Fig. 2 Distribution of features within the W22 genome. The relative density for a variety of features in $1 \mathrm{Mb}$ bins across the maize genome is shown. The purple tracks show the density of genes, LTR transposons and TIR transposons. The relative chromatin accessibility as assessed by MNase (green) and the levels of CG, CHG and CHH methylation (red) are shown. The frequencies of Ds or Mu transposon insertions are also shown (gray).

may be due to reduced levels of RNA-seq data for W22 relative to B73. There are numerous locus-specific differences in splicing observed between B73 and W22 and these are found distributed throughout the genome (Supplementary Fig. 2). One major advantage of sequencing and annotating additional genotypes is the improved ability to analyze high-throughput sequencing data sets. To test the benefit of using a genotype-matched reference, W22 leaf RNA sequencing data (SRA: SRR1986376) was analyzed in parallel using both the W22 and the B73v4 genome references. Mapping RNA-seq reads from W22 seedling leaf tissue to the W22 genome resulted in a mapping rate of $95.7 \%$ while only $91.1 \%$ of these reads could be aligned to the B73v4 genome (Supplementary Fig. 3a). A comparison of the expression level for orthologous genes in B73 and W22 reveals that the expression level estimates vary for a number of genes (Supplementary Fig. 3b). In many cases, these changes in expression were due to altered annotation of the transcripts in W22 relative to B73 (Supplementary Fig. 3c).

The maize genome is largely composed of transposable elements (TEs) ${ }^{28}$. A structural annotation of transposons in the W22v2 genome identified over 177,000 transposons that were classified into 26,833 families (see Methods). Long terminal repeat (LTR) retrotransposons are the most prevalent type of TEs in the W22 genome with 23,144 families accounting for $64 \%$ of the genome. The 10 most abundant LTR families have 1,400-16,395 members and account for $36 \%$ of the genome. Terminal inverted repeat (TIR) DNA transposons were classified into 5 major types and account for $0.46 \%$ of the genome. There are also 23,895 helitron elements that account for $4.6 \%$ of the genome. While many of the TEs are present in intergenic space, there are numerous examples of transposons being annotated within the introns of genes. Nearly $9 \%$ of the FGS genes contain an annotated transposon, including 1,626 genes containing a TIR element and 1,864 genes containing an LTR element. A comparison of the TEs present in the B73 and W22 genome revealed high levels of variation in TE presence and copy number for both TIR and LTR families. There are 937 families of TIR elements that are found in both B73 and W22. Many other TIR families are found only in B73 (107 families) or W22 (62 families) after excluding B73 families that are present only as MITEs. These B73-specific and W22-specific TIR elements are enriched for CACTA (DTC) families (Supplementary Fig. 4a). There are 12,740 families of LTR elements annotated in both B73 and W22 and many other families specific to W22 $(10,531)$ or B73 $(11,032)$. The number of elements in each family is generally similar in the two genomes but there are many examples of strong copy number variation (Supplementary Fig. $4 \mathrm{~b}-\mathrm{d}$ ). However, there are 23 LTR families and 30 TIR families with differences in copy number $>100$ between the two genomes including 27 with higher copy number in W22 and 26 with higher copy number in B73. The analysis of age of LTR elements (based on sequence identity of the two LTRs) in families that are inbred-specific reveals that small inbredspecific families tend to be relatively old and thus may be being purged from the genome while larger inbred specific families tend to be enriched for recent insertion events (Supplementary Fig. 4e). Even in TIR and LTR families with similar copy number we see variation for specific elements. For example, among the 100 TIR families with a single copy in each genome there are 74 examples in which the transposon is inserted into different chromosomes in the two inbred lines. A detailed analysis of native Mutator insertions in the B73 and W22 genomes (see Supplementary Note for details) revealed similar numbers in both genomes (258 in W22 and 259 in B73). However, fewer than half (125) of these insertions are shared in both lines. There are over 100 unique Mutator insertions in each line and $63 \%$ of these are located within $500 \mathrm{bp}$ of an annotated gene. Among the 7,463 LTR families with a single 
copy in both B73 and W22 there are 863 examples in which that single element is located on different chromosomes in the two genomes. These examples of polymorphic TIR and LTR elements highlight the exceptional diversity of TE insertion events among maize genotypes.

Mapping of transposon insertion sites using the W22 genome Significant efforts have been dedicated to the development of stocks with novel transposon insertions in the W22 genetic background. These include several approaches that have mobilized $D s$ elements $^{13}$ and the development a large number of $M u$ insertion stocks ${ }^{7-9}$. A large number of these novel insertions have been sequence indexed to document information on the sequence flanking insertion sites. To date, the insertion sites have often been determined through

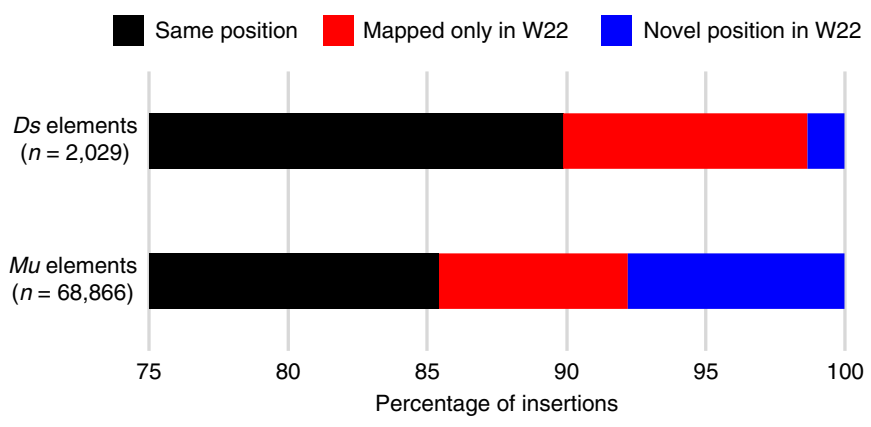

Fig. 3 | Improved ability to document the location of $D$ s and $M u$ insertions using the W22 genome. The $D s(n=2,029)$ and $M u(n=68,866)$ insertion flanking sequences were mapped to the W22 genome. The proportion of insertion sites that mapped to the same location (black), different locations (blue) or only to W22 (red) was determined. mapping of flanking sequences to the B73 genome. However, a large number of insertions could not be accurately placed due to insufficient sequence identity with B73. To precisely position the $2,029 \mathrm{Ds}$ and 68,866 Mu insertions that have been generated, sequences adjacent to the transposon were mapped against the W22v2 genome. This provided novel insertion sites for $12 \%-15 \%$ of these insertions (Fig. 3). For the 13,444 UniformMu stocks, the mapping of flanking sites to the W22 genome provided novel insertion site information for over 10,000 insertions and increased the properly placed insertions from 4.35 per line to 5.1 per line. Furthermore, the genomic distributions of $D s$ and $M u$ insertions are distinct from one another (Fig. 2), indicating complementarity of these resources for functional genomics studies. The improved mapping resolution of these insertions coupled with a better resolution of gene content and position afforded by W22v2 assembly will greatly increase the utility of the $D s$ and $M u$ reverse genetics resources. In particular, the $D s$ resource is designed for remobilization of elements to flanking regions of the genome, so accurate placement of insertions to gene models and local gene content is imperative for efficient mutagenesis. Similarly, better gene models will enable a more accurate prediction of phenotypic consequences of transposon insertions, particularly those that map to the $5^{\prime}$ and $3^{\prime}$ ends of a gene and thus may induce subtle mutant phenotypes.

\section{Ds and $M u$ probe different compartments of the genome}

$D s$ and $M u$ represent two different transposon superfamilies (DTA and DTM). Previous studies have suggested differences in terms of the frequency of linked transposition and insertion locations relative to genes ${ }^{13,29,30}$. Several properties of chromatin in the W22 genome were analyzed to better understand the factors that influence the insertion sites for $\mathrm{Ds}$ and $\mathrm{Mu}$. The context-specific DNA methylation patterns throughout the W22 genome were documented using whole-genome bisulfite sequencing (WGBS) and chromatin accessibility was assessed using MNase treatments (Figs. 2 and $4 \mathrm{a}-\mathrm{c}$ ).
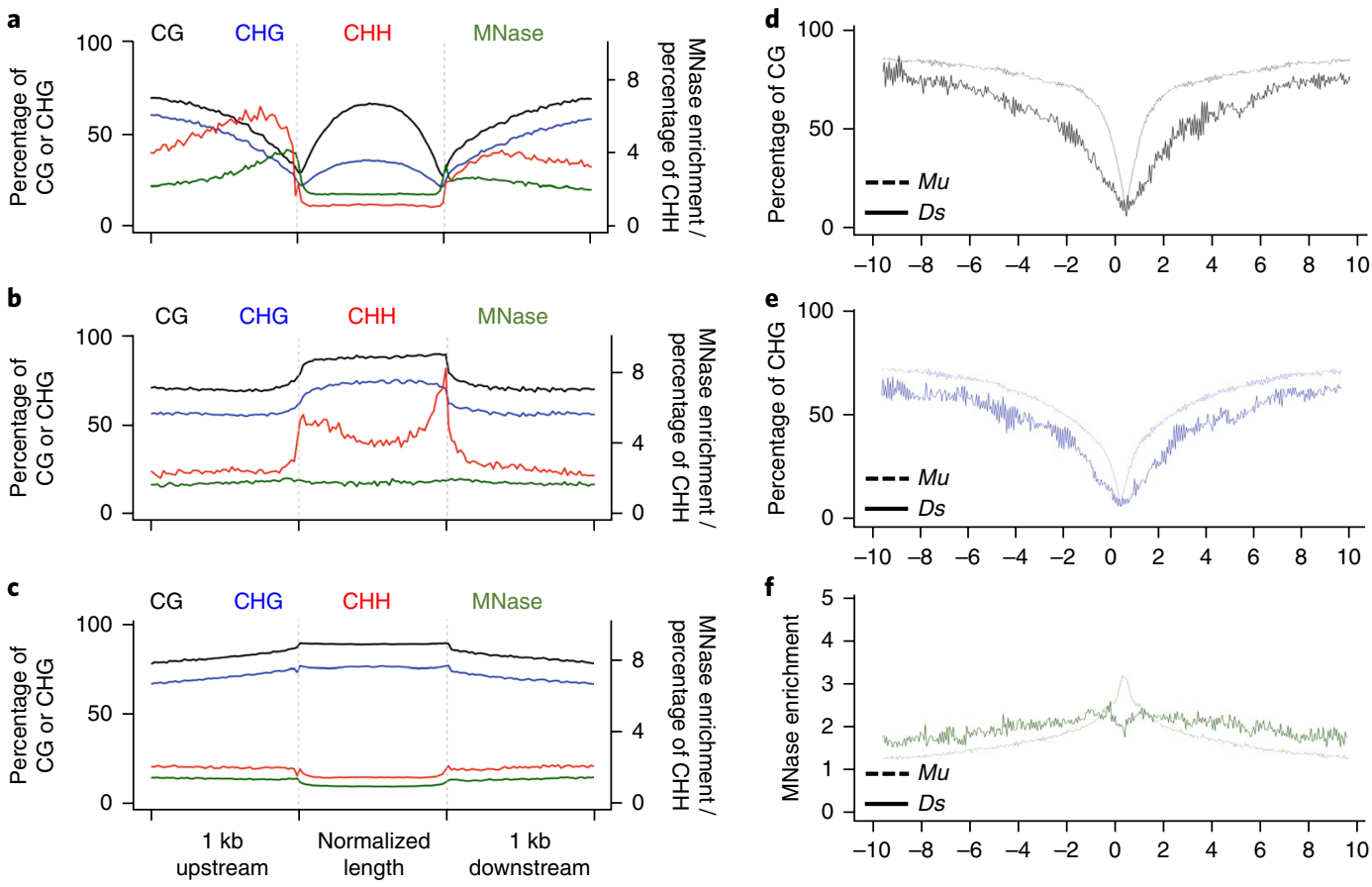

Fig. 4 | Patterns of chromatin over maize genes and transposons. a-c, The levels of CG methylation (black), CHG methylation (blue) or CHH methylation (red) over the $1 \mathrm{~kb}$ flanking regions and maize genes (a), TIR transposons (b) or LTR transposons (c). In addition, the enrichment for open chromatin (green) based on MNase digests is shown for the same regions. $\mathbf{d - f}$, Chromatin profiles for sites targeted by Ds (solid lines) or Mu (dashed lines). The relative levels of CG methylation (d) and CHG methylation (e) or open chromatin (f) are shown for the flanking regions (up to $10 \mathrm{~kb}$ ) near Ds or Mu insertion sites. 


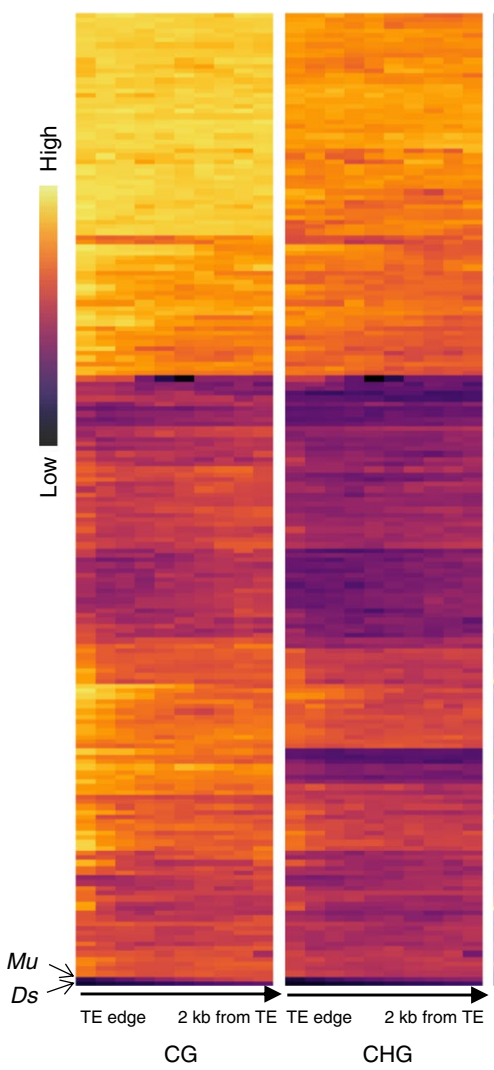

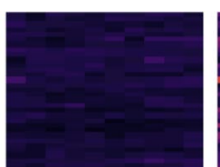

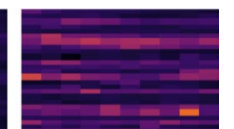

b

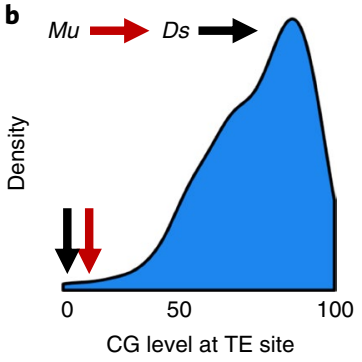

c
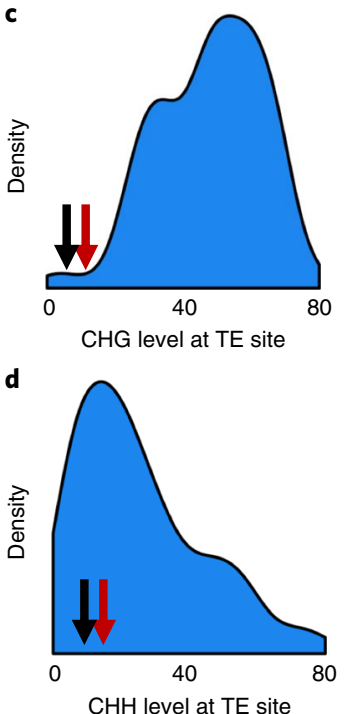

Fig. 5 | Chromatin profiles for regions flanking pre-existing TIR elements or novel Ds or Mu insertion sites. a, For 191 TIR families with at least 20 elements present in the W22 genome, we determined the relative level of CG and CHG methylation in 100-bp windows for the 1-kb flanking regions. These levels of methylation were used to cluster the families and a heatmap allows visualization of the patterns of DNA methylation for flanking regions. $\mathbf{b}, \mathbf{c}$, The distributions of the average level of methylation for $\mathrm{CG}(\mathbf{b})$ or $\mathrm{CHG}$ (c) and $\mathrm{CHH}$ (d) contexts in the 100 bp surrounding each TE family are shown as density plots. The levels of methylation at sites where $\mathrm{Mu}$ (red arrows) or Ds (black arrows) elements can insert are shown as arrows.

The levels and genomic distribution of DNA methylation in W22 are similar to patterns observed in other maize inbred lines ${ }^{31-33}$. Open chromatin and $\mathrm{CHH}$ methylation are substantially enriched in promoter regions and following the transcription termination sites of annotated genes (Fig. 4a). In contrast, CHG and CG methylation are relatively depleted in these regions (Fig. 4a). CG and CHG methylation are enriched within TIR and LTR transposons (Fig. 4b,c). CHH methylation is enriched at TIR elements but shows minimal enrichment at LTR elements. Chromatin accessibility does not vary substantially within or near transposons in the W22 genome (Fig. 4b,c).

The locations for novel transposon insertions can be quite sensitive to chromatin structure ${ }^{34}$. The profiles of open chromatin and DNA methylation were assessed for the regions flanking potential $D s$ and $M u$ insertion sites (Fig. $4 \mathrm{~d}-\mathrm{f}$ and Supplementary Fig. 5). There are differences in the accessibility of chromatin at $M u$ and $D s$ insertion sites. The $M u$ insertion sites are marked by an increase in chromatin accessibility while $D s$ insertion sites do not vary substantially compared to flanking regions. Both $\mathrm{Mu}$ and $D$ s prefer to insert in regions with very low CG and CHG methylation. A metaprofile from bisulfite sequencing reveals that $M u$ insertion sites are often very near regions with CG or CHG methylation as both of these modifications are present on either side of the insertion site. In contrast, the increase in CG or CHG methylation near $D s$ insertion sites tends to occur further away from the insertion site. $\mathrm{CHH}$ methylation tends to be enriched at regions immediately adjacent to $M u$ insertion sites but does not show strong patterns near Ds insertion sites (Supplementary
Fig. 5). These patterns could explain the preference for $\mathrm{Mu}$ to insert in the $5^{\prime}$ and $3^{\prime}$ UTR regions of genes while $D s$ is more likely to insert within coding regions.

The W22 genome contains a number of native TIR transposon families. The patterns of chromatin within these TIR elements include elevated DNA methylation and relatively closed chromatin (Fig. 4b). The chromatin at the regions flanking these TIR elements likely reflects endogenous genomic information that influences chromatin state as well as the influences of the TE on neighboring chromatin. Given the differences in chromatin profiles for the insertion sites of $D s$ or $M u$, we were interested in comparing the chromatin at these regions with the chromatin flanking pre-existing TIR insertions. We collected chromatin profiles for $2 \mathrm{~kb}$ of flanking regions for all TIR families with $>50$ elements and clustered these profiles together with the profile of chromatin flanking potential $D s$ and $M u$ insertion sites (Fig. 5). There are several quite distinct patterns of chromatin flanking TIR elements. Some families show very high methylation for flanking regions while other families are more likely to have low levels of CG and CHG methylation. The $M u$ and $D s$ potential insertion sites are outliers for the levels of CG and CHG methylation compared to the regions flanking pre-existing element insertion sites. The analysis of the methylation levels for the first $100 \mathrm{bp}$ at the edge of the TE reveals that both $M u$ and $D s$ insertion sites have much lower levels of CG and CHG methylation than observed for pre-existing TIR insertion sites (Fig. 5b,c). In contrast, the levels of $\mathrm{CHH}$ methylation near $\mathrm{Mu}$ and $\mathrm{Ds}$ potential insertion sites are very similar to the levels of $\mathrm{CHH}$ methylation at pre-existing TIR insertion sites (Fig. 5d). 
Since the turn of the twentieth century, maize has provided a model system for genetic research ${ }^{35}$. The development of a colorconverted W22 inbred enabled high-resolution mapping of numerous traits influencing anthocyanin and flavonoid accumulation and led to the adoption of W22 as the workhorse of maize genetics ${ }^{1-4}$. In recent years the genomics data sets generated from studies of transposon insertion alleles have been analyzed in the context of the B73 reference genome. Numerous studies have documented the widespread genetic variation among inbred genotypes of maize including SNPs, copy number variation and indels ${ }^{15,19,36,37}$. The availability of a high-quality assembly and annotation for W22 will increase the resolution of studies of mutant alleles in this genetic background and recent improvements in maize transformation are likely to enable higher efficiencies of W22 transformation ${ }^{38}$. Thus, W22 is well positioned to serve as the maize reference inbred for functional genomics studies.

The annotation of TEs present in the W22 and B73 genome will provide new opportunities to study the sources, and effects, of TE polymorphisms among maize lines. While there are many shared transposons in B73 and W22, there are numerous examples of severe copy number and presence/absence variation of certain families. There are also many examples of polymorphic TE insertions within or near genes that could influence regulation of these genes. Locus-specific studies have provided evidence for the importance of polymorphic TE insertions that influence the regulation of nearby genes, resulting in quantitative trait loci affecting important agronomic traits ${ }^{39-41}$. The ability to have a high structural resolution of both genomes will serve as a resource to enable detailed studies of how transposons influence gene expression among diverse maize genotypes. The knowledge of complex haplotype variation can also be used to inform pan-genomebased approaches that incorporate rearrangements and insertion/deletions from large-scale resequencing studies to augment SNP-based GWAS studies.

The availability of epigenome profiles for DNA methylation and chromatin accessibility provides an opportunity to study chromatin profiles for thousands of $M u$ and $D s$ insertion sites. The differences in these profiles suggest that $M u$ and $D s$ utilize distinct targeting mechanisms and occupy different niches of the W22 genome. Mu elements tend to insert in small windows of accessible chromatin that are near highly methylated regions. Previous work has noted the propensity for $M u$ elements to insert near gene promoters and these regions likely contain the chromatin profiles noted for $\mathrm{Mu}$ elements $^{29,30}$. In contrast, $D s$ frequently inserts into regions with low CG and CHG methylation that are not enriched for accessible chromatin. This is compatible with the concept of preferential insertion of $D s$ elements within the coding regions of maize genes $^{13}$. Interestingly, chromatin profiles for random sites selected within gene promoters or coding regions do not match the profiles observed for $M u$ or $D s$ sites. This suggests that $M u$ and $D s$ have specific targeting beyond simple selection for promoters or coding regions. Furthermore, the chromatin profiles at loci targeted by active $M u$ or $D s$ elements are distinct from the chromatin profiles at sites that flank inactive pre-existing insertion sites. This likely reflects the fact that many of these pre-existing element insertion sites are targeted for silencing and the chromatin properties of the elements themselves can spread into the flanking regions. It is possible that many of the pre-existing TIR families might insert into regions with chromatin profiles similar to $M u$ or $D s$ if they were active. As additional high-quality maize genome assemblies become available, comparative analysis of the maize pan-genome is likely to provide further insight into how transposons have shaped the maize genome through the course of domestication.

Received: 19 October 2017; Accepted: 17 May 2018;

Published online: 30 July 2018

\section{References}

1. Brink, R. A., Styles, E. D. \& Axtell, J. D. Paramutation: directed genetic change. Paramutation occurs in somatic cells and heritably alters the functional state of a locus. Science 159, 161-170 (1968).

2. Mertz, E. T., Bates, L. S. \& Nelson, O. E. Mutant gene that changes protein composition and increases lysine content of maize endosperm. Science 145, 279-280 (1964).

3. Kermicle, J. L. Dependence of the R-mottled aleurone phenotype in maize on mode of sexual transmission. Genetics 66, 69-85 (1970).

4. Fedoroff, N. V., Furtek, D. B. \& Nelson, O. E. Cloning of the bronze locus in maize by a simple and generalizable procedure using the transposable controlling element Activator (Ac). Proc. Natl Acad. Sci. USA 81, 3825-3829 (1984)

5. Chen, J., Greenblatt, I. M. \& Dellaporta, S. L. Transposition of Ac from the P locus of maize into unreplicated chromosomal sites. Genetics 117, 109-116 (1987).

6. Dooner, H., English, J., Ralston, E. \& Weck, E. A single genetic unit specifies two transposition functions in the maize element activator. Science 234, 210-211 (1986).

7. McCarty, D. R. et al. Steady-state transposon mutagenesis in inbred maize. Plant J. 44, 52-61 (2005).

8. McCarty, D. R. et al. Mu-seq: sequence-based mapping and identification of transposon induced mutations. PLoS One 8, e77172 (2013).

9. Liu, P., McCarty, D. R. \& Koch, K. E. Transposon mutagenesis and analysis of mutants in UniformMu maize (Zea mays). Curr. Protoc. Plant Biol. 1, 451-465.

10. Brutnell, T. P. \& Conrad, L. J. Transposon tagging using Activator (Ac) in maize. Methods Mol. Biol. 236, 157-176 (2003)

11. Kolkman, J. M. et al. Distribution of Activator (Ac) throughout the maize genome for use in regional mutagenesis. Genetics 169, 981-995 (2005).

12. Li, Y., Segal, G., Wang, Q. \& Dooner, H. K. Gene tagging with engineered Ds elements in maize. Methods Mol. Biol. 1057, 83-99 (2013).

13. Vollbrecht, E. et al. Genome-wide distribution of transposed Dissociation elements in maize. Plant Cell 22, 1667-1685 (2010).

14. Springer, N. M. et al. Maize inbreds exhibit high levels of copy number variation $(\mathrm{CNV})$ and presence/absence variation $(\mathrm{PAV})$ in genome content. PLoS Genet. 5, e1000734 (2009).

15. Lai, J. et al. Genome-wide patterns of genetic variation among elite maize inbred lines. Nat. Genet. 42, 1027-1030 (2010).

16. Fu, H. \& Dooner, H. K. Intraspecific violation of genetic colinearity and its implications in maize. Proc. Natl Acad. Sci. USA 99, 9573-9578 (2002).

17. Hirsch, C. N. et al. Insights into the maize pan-genome and pantranscriptome. Plant Cell 26, 121-135 (2014).

18. Jiao, Y. et al. Improved maize reference genome with single-molecule technologies. Nature 546, 524-527 (2017).

19. Hirsch, C. N. et al. Draft assembly of elite inbred line $\mathrm{PH} 207$ provides insights into genomic and transcriptome diversity in maize. Plant Cell 28, 2700-2714 (2016).

20. Zheng, G. X. Y. et al. Haplotyping germline and cancer genomes with high-throughput linked-read sequencing. Nat. Biotechnol. 34, 303-311 (2016).

21. Luo, M.-C. et al. Optical nano-mapping and analysis of plant genomes. Methods Mol. Biol. 1429, 103-117 (2016).

22. Lu, F. et al. High-resolution genetic mapping of maize pan-genome sequence anchors. Nat. Commun. 6, 6914 (2015).

23. Dooner, H. K. \& He, L. Maize genome structure variation: interplay between retrotransposon polymorphisms and genic recombination. Plant Cell 20, 249-258 (2008)

24. Simão, F. A., Waterhouse, R. M., Ioannidis, P., Kriventseva, E. V. \& Zdobnov, E. M. BUSCO: assessing genome assembly and annotation completeness with single-copy orthologs. Bioinformatics 31, 3210-3212 (2015).

25. Swanson-Wagner, R. A. et al. Pervasive gene content variation and copy number variation in maize and its undomesticated progenitor. Genome Res. 20, 1689-1699 (2010)

26. McCormick, R. F. et al. The Sorghum bicolor reference genome: improved assembly, gene annotations, a transcriptome atlas, and signatures of genome organization. Plant J. 93, 338-354 (2018).

27. Mei, W. et al. A comprehensive analysis of alternative splicing in paleopolyploid maize. Front. Plant Sci. 8, 694 (2017).

28. Schnable, P. S. et al. The B73 maize genome: complexity, diversity, and dynamics. Science 326, 1112-1115 (2009).

29. Dietrich, C. R. et al. Maize Mu transposons are targeted to the $5^{\prime}$ untranslated region of the gl8 gene and sequences flanking $\mathrm{Mu}$ target-site duplications exhibit nonrandom nucleotide composition throughout the genome. Genetics 160, 697-716 (2002)

30. Liu, S. et al. Mu transposon insertion sites and meiotic recombination events co-localize with epigenetic marks for open chromatin across the maize genome. PLoS Genet. 5, e1000733 (2009).

31. Gent, J. I. et al. CHH islands: de novo DNA methylation in near-gene chromatin regulation in maize. Genome Res. 23, 628-637 (2013). 
32. Regulski, M. et al. The maize methylome influences mRNA splice sites and reveals widespread paramutation-like switches guided by small RNA. Genome Res. 23, 1651-1662 (2013).

33. Li, Q. et al. Examining the causes and consequences of context-specific differential DNA methylation in maize. Plant Physiol. 168, 1262-1274 (2015).

34. Sultana, T., Zamborlini, A., Cristofari, G. \& Lesage, P. Integration site selection by retroviruses and transposable elements in eukaryotes. Nat. Rev. Genet. 18, 292-308 (2017).

35. Nannas, N. J. \& Dawe, R. K. Genetic and genomic toolbox of Zea mays. Genetics 199, 655-669 (2015).

36. Messing, J. \& Dooner, H. Organization and variability of the maize genome. Curr. Opin. Plant Biol. 9, 157-163 (2006).

37. Chia, J. M. et al. Maize HapMap2 identifies extant variation from a genome in flux. Nat. Genet. 44, 803-807 (2012).

38. Altpeter, F. et al. Advancing crop transformation in the era of genome editing. Plant Cell 28, 1510-1520 (2016).

39. Studer, A., Zhao, Q., Ross-Ibarra, J. \& Doebley, J. Identification of a functional transposon insertion in the maize domestication gene tb1. Nat. Genet. 43, 1160-1163 (2011).

40. Yang, Q. et al. CACTA-like transposable element in ZmCCT attenuated photoperiod sensitivity and accelerated the postdomestication spread of maize. Proc. Natl Acad. Sci. USA 110, 16969-16974 (2013).

41. Castelletti, S., Tuberosa, R., Pindo, M., \& Salvi, S. A MITE transposon insertion is associated with differential methylation at the maize flowering time QTL Vgt1. G3 4, 805-812 (2014).

\section{Acknowledgements}

This work was supported by grants from the following: National Science Foundation NSF-MCB 0920218 to H.K.D.; NSF-MCB 1025976 to K.E.K.; NSF-IOS 1031416 to A.M.S.; NSF-IOS 1237931 to N.M.S.; NSF-IOS 1116561 to D.R.M. and K.E.K.; NSF-IOS 1238014 to E.S.B.; NSF-IOS 1314143 to T.P.B. and E.V.; NSF IOS-1339237 and IOS1139329 to G.J.; NSF MCB-1412218 to A.M.S. and W.B.B.; NSF IOS-1444514 to R.K.D. NSF-IOS 1521100 to J.-C.G., K.E.K. and D.R.M.; NSF-IOS 1547787 to W.B.B.; NSF Graduate Research Fellowship and University of Florida Graduate Student Fellowship to C.G.; NSF Graduate Research Fellowship DGE-0802270 to C.G.; the United States Department of Agriculture (USDA) in kind to C.M.A., E.K.S.C., M.R.W. and J.L.P.; USDA-ARS 6036-21000-011-00D to C.T.H.; USDA-NIFA 2016-67013-24747 to N.M.S. USDA-NIFA 2011-67003-30215 to D.R.M. and K.E.K.; USDA-NIFA 2011-67013-30082 to D.R.M. and M.S.; USDA-ARS and the NSF-IOS Plant Genome 1238014 to E.S.B.; the Enterprise Rent-A-Car Institute for Renewable Fuels at the Donald Danforth Plant Science Center to T.P.B.; and the Vasil-Monsanto Endowment to A.M.S. The use of trade names, commercial products or corporations in this publication is for the information and convenience of the reader and does not imply an official recommendation, endorsement or approval by the US Department of Agriculture or the Agricultural Research Service for any product or service to the exclusion of others that may be suitable. USDA is an equal opportunity provider and employer.

\section{Author contributions}

K.R.A., F.B., H.K.D., D.M.-J., T.K., J.S. and J.T.V. provided or isolated biological materials; O.B., K.B., G.B.-Z., G.K., G.R., D.S.-T. and I.S. performed the genome assembly; O.B., K.B., R.B., G.B.-Z., E.S.B., R.K.D., L.H.D., C.D., G.K., F.L., G.R., D.S.-T., E.V., J.S. and I.S. performed quality assessments and assembly validation; M.S.C., G.J., Y.J., T.G.K., J.C.S., M.C.S., D.W., S.W. and M.R.W. annotated the genome; C.M.A., E.K.S.C., K.A.E. and J.L.P. developed visualization tools; S.N.A., F.B., W.B.B., J.-C.G., R.D., C.G., K.E.K., T.K., D.M.-J., W.M., D.R.M., A.M.S. and M.S. performed transcriptome analyses; S.N.A., K.R.A., C.D., C.T.H., K.E.K., D.R.M., J.M.N., M.C.S., E.V., K.W. and W.X. mapped and characterized TE distribution; H.W.B., Q.L., J.M.N. and D.L.V. characterized epigenetic profiles; and T.P.B., P.C., H.K.D. and N.M.S. contributed to experimental design and wrote the manuscript.

\section{Competing interests}

Several authors are employed by companies that provide genome sequencing and assembly support. O.B., K.B., G.B.-Z., P.C., G.K., G.R., D.S.-T. and I.S. are employed by NRGene and J.S. is employed by BioNano Genomics.

\section{Additional information}

Supplementary information is available for this paper at https://doi.org/10.1038/ s41588-018-0158-0.

Reprints and permissions information is available at www.nature.com/reprints. Correspondence and requests for materials should be addressed to T.P.B. Publisher's note: Springer Nature remains neutral with regard to jurisdictional claims in published maps and institutional affiliations.

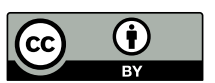

Open Access This article is licensed under a Creative Commons Attribution 4.0 International License, which permits use, sharing, adaptation, distribution and reproduction in any medium or format, as long as you give appropriate credit to the original author(s) and the source, provide a link to the Creative Commons license, and indicate if changes were made. The images or other third party material in this article are included in the article's Creative Commons license, unless indicated otherwise in a credit line to the material. If material is not included in the article's Creative Commons license and your intended use is not permitted by statutory regulation or exceeds the permitted use, you will need to obtain permission directly from the copyright holder. To view a copy of this license, visit http://creativecommons.org/licenses/by/4.0/. 


\section{Methods}

W22 genome sequencing and assembly. DNA sequencing. Genomic DNA was isolated from 10-day-old W22 seedling tissues and nuclear DNA was purified and fractionated from organellar DNA on a $\mathrm{CsCl}$ gradient ${ }^{42}$. A genomic library of 800-bp DNA fragment sizes was prepared using the TruSeq DNA Sample Preparation Kit version 2 according to the manufacturer's protocol (Illumina) A second shotgun library was made using the same kit from DNA template fragments size selected of $\sim 450 \mathrm{bp}$ with no PCR amplification (PCR-free). This fragment size was designed to produce a sequencing overlap of the fragments to be sequenced on the HiSeq2500 v2 Rapid mode as PE sequencing 250 nucleotides per end, thus creating an opportunity to produce 'stitched' reads of approximately $250 \mathrm{bp}$ to $480 \mathrm{bp}$ in length. Multiple MP libraries were prepared with the objective to increase sequence diversity and genome coverage including three size classes $(2-4 \mathrm{~kb}, 5-7 \mathrm{~kb}$ and $8-10 \mathrm{~kb})$ using the Illumina Nextera Mate-Pair Sample Preparation Kit (Illumina). The 800-bp shotgun library and the MP libraries were sequenced on an Illumina HiSeq2500 as PE with 160-bp read lengths for each of the fragments using the SBS sequencing kit version 4 . Each library was sequenced to a coverage of $\sim 65 \mathrm{~Gb}(\sim 30 \times$ the maize genome size $)$. The 450 -bp PCR-free shotgun library was sequenced on an Illumina HiSeq2500 using Rapid mode with v2 chemistry as PE with 250-bp read lengths. This library was sequenced to a coverage of $\sim 130 \mathrm{~Gb}(\sim 60 \times$ the maize genome size). In total, $\sim 180 \times$ of sequencing data was produced for this project. All sequencing was conducted at the Roy J. Carver Biotechnology Center (Urbana, IL) at the University of Illinois.

Genome assembly. The W22 genome was assembled using DenovoMAGIC 2 (NRGene). Several previous studies have described this assembly approach ${ }^{19,43}$ and we provide a summary of the implementation of this approach for W22. Read pre-processing included removal of PCR duplicates, Illumina adaptor AGATCGGAAGAGC and Nextera linkers (for MP libraries). For the $2 \times 250450$-bp $\mathrm{PE}$ libraries, overlapping reads were merged with a minimal required overlap of $10 \mathrm{bp}$ to create stitched reads. Error correction of pre-processed reads was performed by scanning to detect reads with putative sequencing errors (containing a sub-sequence that does not reappear several times in other reads) and those reads were filtered out. The first step of de novo assembly consisted of building a de Bruijn graph $(k$-mer $=239 \mathrm{bp})$ of contigs from the 'stitched' reads. The large $k$-mer length significantly reduced the complexity of the de Bruijn graphs, which is essential for high-quality assembly of very complex genomes. No bubble merge and no repeat masking filtering were used.

To scaffold the contigs of the de Bruijn graph, non-repetitive contigs within the graph were identified and assembled into scaffolds based on mapping information of the 'stitched' reads. Scaffolding was completed using a directed graph containing scaffolds longer than $200 \mathrm{bp}$ as nodes, and edges were based on the PE and MP links as vertices. Erroneous connections were filtered out to generate unconnected sub-graphs that were ordered into scaffolds. PE reads were used to find reliable paths in the graph for additional repeat resolving. This was accomplished through searching the de Bruijn graph for a unique path connecting pairs of reads mapping to two different scaffolds. The scaffolds were then furthered ordered and linked using the MP libraries, estimating gaps between the contigs according to the distance of MP links. Linking scaffolds with MP reads required confirmation of at least three filtered MPs or at least one filtered MP with supporting confirmation from two or more filter-failed MPs where the Nextera adaptor was not found. Scaffolds shorter than $200 \mathrm{bp}$ were masked and links between non-repetitive contigs mapping to the same scaffolds were united, generating a directed scaffold graph. Further ordering of scaffolds was achieved through alignment of the scaffolds to the B73 reference genome (RefGen_v2) and selection of the most probable genomic location. We did not use the B73 reference to create the W22 scaffolds. The scaffolds were then placed into the pseudomolecules to maximize linear synteny between the assembled and B73 genomes. To improve the accuracy of our scaffolds, we used an independent method to identify and correct misassemblies. We sequenced one HiSeq X10 lane (total of $120 \mathrm{G}$ of $150 \times 2 \mathrm{bp}$ raw sequencing data) with one long-range barcoded DNA library (Chromium system by 10x genomics). Reads were mapped to the assembled scaffolds and clusters of reads with the same barcode mapped to adjacent contigs in the scaffolds were identified. Overall, we detected 22 million clusters and the estimated length of $>2$ million of those molecules is above $50 \mathrm{~kb}$ (the estimated average length of all molecules is $15 \mathrm{~kb}$ with s.d. $=23 \mathrm{~kb}$ ). Next we scanned along each scaffold with a 20 -kb-length window and counted: the number of distinct clusters that cover the left edge of the window; the number of distinct clusters that cover the right edge of the window; and the number of distinct clusters that cover the entire window indicating a support for this $20 \mathrm{~kb}$ connection by several long molecules (identified by having distinct barcodes). On average, 255 long molecules (s.d. $=74$ ) cover each edge of the window and 124 long molecules support an entire window (s.d. $=31$ ). There are 34 potential chimeric scaffolds that were detected as windows with a statistically significant (using a hyper-geometric statistical test with a Bonferroni correction, $P$ value $2.5 \times 10^{-7}$ ) lower number of supporting overlapping long molecules. Twenty-three of these potential chimeric scaffolds were also detected in the genetic markerbased scaffold validation stage (see below). These chimeric scaffolds were broken and reassembled in $\mathrm{W} 22 \mathrm{v} 2$ using the X10 information.
Bionano mapping and assembly validation. High-molecular-weight genomic DNA was isolated from $0.5 \mathrm{~g}$ of W22 leaf tissue using the IrysPrep Plant Tissue DNA Isolation Kit (RE-014-05). The DNA was labeled at Nt.BspQI sites using the IrysPrep NRLS labeling kit (RE-012-10). Molecules were collected using BioNano IrysChips at $100 \times$ coverage with an average molecule length of $220 \mathrm{~kb}$. The data were then de novo assembled using 'optArgument human' The resulting W22 assembly contained 1,872 BioNano genome maps (equivalent to contigs) that span a total of $2,171 \mathrm{Mb}$ with an N50 of $1.646 \mathrm{Mb}$. After assembly, the W22v2 genome maps were aligned to an in silico BspQI-digested cmap of the NRGene sequence assembly. The final alignment parameter 'Total Unique Aligned Len/ Ref Len' was 0.983 , indicating that $98.3 \%$ of the BioNano maps aligned uniquely with the NRGene assembly. As a means to directly compare the quality of the W22 assembly with the quality of the B73 assembly ${ }^{18}$, we created a hybrid scaffold that incorporated both the sequence and Bionano data. The W22 hybrid assembly contained $97.4 \%$ of the Bionano contigs and $99.4 \%$ of the sequence assembly. These values are slightly better than equivalent values for B73 (95.1\% and 98.4\%, respectively), indicating that the ordering and orientation of contigs in the NRGene assembly are on par with the PacBio-based B73 reference assembly.

Scaffold validation. The 4.4 million high-resolution genetic anchors, which were developed from $>14,000$ maize inbred lines ${ }^{22}$, were used to validate the scaffolds of the W22 assembly. The sequence anchors were aligned to an initial assembly of W22 using Bowtie2. The scaffolds with at least 20 mapped anchors were examined for the consistency of sequence assembly and genetic position. A total of 409 scaffolds with an overall length of $2.17 \mathrm{~Gb}$ were evaluated. We found that 23 scaffolds were assembly artifacts, which were then corrected in the W22v2 assembly.

W22 genome annotation. W22 gene annotation. Annotation of protein-coding genes was performed using MAKER-P pipeline software ${ }^{44}$, with parameters and evidence similar to those recently used to annotate B73 $3^{18,45}$. Repeat masking ('RepeatMasker Home Page' 2017) was performed using exemplar transposon sequences ${ }^{28}$ available online at the maize TE database ('Maize Database' 2017). We excluded helitron and MULE elements to avoid false-positive masking from captured exon sequences in such elements. Gene expression evidence included PacBio Iso-seq long reads sequenced from cDNA libraries of six tissues in B73 $(n=111,151)^{46}$. In addition, we included the following transcriptome assemblies, each processed to exclude short transcripts $(<300 \mathrm{bp})$ and redundancies based on application of CD-HIT ${ }^{47}$ : a pooled set of 94 transcriptome assemblies constructed from publicly available RNA-seq reads $(n=508,233)^{45}$; a transcriptome assembly of B73 seedlings $(n=112,963)^{48}$; a transcriptome assembly of W22 tissues $(n=589,743)$. Cross-species evidence was supplied in the form of the following annotated protein files downloaded from Gramene release $46\left(\right.$ Gramene FTP ${ }^{49}$ : Arabidopsis_thaliana.TAIR10.27.pep.all.fa;Brachypodium_distachyon.v1.0.27.pep all.fa; Oryza_sativa.IRGSP-1.0.27.pep.all.fa; Setaria_italica.JGIv2.0.27.pep.all.fa; and Sorghum_bicolor.Sorbi1.27.pep.all.fa. Alignment and downstream processing of sequence evidence to the repeat-masked W22 reference was performed within the MAKER-P pipeline using default parameters. For gene model prediction, the pipeline incorporated AUGUSTUS ${ }^{50}$ applied with the 'maize5' model and FGENESH ${ }^{51}$ applied with the 'monocot' model. Stable gene identifiers were assigned using the format $\mathrm{Zm00004bXXXXXX} \mathrm{(where} \mathrm{the} \mathrm{Xs} \mathrm{represent} \mathrm{a} \mathrm{random}$ 6-digit number), as specified under 'A Standard For Maize Genetics Nomenclature' available at MaizeGDB ('Maize Genetics Nomenclature' 2017). For further details on the characterization and screening of gene models, see the Supplementary Note.

TE annotation. To identify structurally intact copies of TEs in the genome assembly, we followed a similar approach to that used for B73v4 $4^{18}$ to characterize features such as target site duplications and terminal motifs. To encourage consistency of nomenclature, we applied the 80-80-80 bioinformatic cutoffs ${ }^{52}$, to match W22 TE copies to existing families in B73 based on $80 \%$ identity across $80 \%$ of the length of the TE or its functional sequence, of at least 80 base pairs. For TEs that could not be assigned to a family present in B73, we generated new family names. Each TE is given a unique identifier in the form RLG00001Zm00004b00001, using the Zm00004b identifier for W22 assigned by the maize community nomenclature committee for gene names. This reflects the TE superfamily (RLG, retrotransposon, LTR, Gypsy), a five digit family number (00001), the W22 identifier (Zm00004b) and a unique identifier for this TE copy (00001). For LTR elements, we apply an iterative search of LTRharvest ${ }^{53}$, removing previously identified copies from the genome, and rerunning LTRharvest with the reduced genome. For TIR elements, we utilize TARGeT ${ }^{54}$, using TE models from the maize TE consortium (MTEC), as well as novel structural families identified using detectMITE ${ }^{55}$. For helitrons, we use the terminal $30 \mathrm{bp}$ at the $3^{\prime}$ end to assign to B73 families, and generate new families as needed. For SINE elements, we use SINEfinder ${ }^{56}$, and for LINE elements, we use TARGeT on MTEC TE models. The TE detection pipeline and associated files can be found at github.com/ mcstitzer/w22_te_annotation.

Characterization of variation in gene content and arrangement. To compare syntenic ortholog retention between W22 and B73, we queried the unmasked W22 genome against the unmasked B73 version 4 from Ensembl release 36 and the outgroup 
Sorghum bicolor v3.126 using CoGe's SynFind (lastz, 50-gene window, minimum of 5 syntelogs per window), which compares synteny across multiple genomes at once, and then reciprocally compared B73 against W22 and Sorghum.

Determining locally duplicated genes. Locally duplicated genes were determined using a method similar to that used to determine the number of shared genes between W22 and B73. The CDS sequences from W22 AGPv2 and B73 AGPv4 genomes were each searched against themselves using COGE's SynFind tool ${ }^{57}$, and the resulting unfiltered LAST search results were downloaded. In each search, LAST hits were cleaned by: removing hits with an e-value of 0.001 or more; condensing hits from multiple transcripts between gene models, to a single hit between gene models (that is, a hit between two gene models was retained if at least one transcript model from each gene model had a valid hit to each other); and removing hits between gene models that are not on the same chromosome. The remaining non-self LAST hits were treated as mappings between paralogs within each inbred genome and were used to determine locally duplicated genes. Distances between genes in the remaining LAST hits were determined by calculating their order and orientation on the chromosome and determining the number of intervening genes. Any gene pair that had less than a maximum of 20 intervening genes was classified as locally duplicated ${ }^{58}$.

Shared and unique tandem duplicates in B73 and W22 were determined by using the master list of ortholog mappings using SynFind. Reciprocal SynFind searches were used where W22 was searched against B73 and vice versa. The reciprocal B73 to W22 gene model mappings that were found in both searches were filtered as syntenic orthologs between the two genomes. Tandem duplications were a subset of the locally duplicated genes that had no intervening genes between them.

Analysis of gene expression. RNA-seq alignments. RNA-seq data for W22 leaf (SRA: SRR1986376) were aligned to both the W22 and B73v4 reference genomes. Reads were trimmed using Cutadapt version 1.8.1 and mapped using tophat2 version 2.0.13 to both genomes. Stranded reads were assigned to gene models for each reference using HTSeq version 0.5.3. The counts per gene were normalized to reads per million mapped reads. To compare gene expression values for mappings to the two references, homologus genes were determined from predicted protein sequences for the first isoform of each gene using OrthoFinder version 1.1.8. Only genes with a 1-to-1 match between W22 and B73v4 were retained for comparison.

Alternative splicing analysis. W22 RNA-seq reads were trimmed using Cutadapt 1.7.1 to remove adapters and low quality sequences trimmed using Trimmomatic 0.32. Processed reads were then aligned to the genome using STAR 2.5.2 and two pass alignment steps. Sixty-seven RNA-seq sample were aligned to STAR individuals, and splice junction from 67 samples were combined together and only splice junction $>=3$ reads in at least one sample kept. These remaining splice junctions were fed into STAR as guidance and the second alignment was run. Duplicate reads were detected and removed using Picard 2.10.3 MarkDuplicates. Two approaches, Stringtie 1.3.3 and Cufflinks 2.2.1, were used for the genome guided assembly; in addition, we also used de novo transcripts from Trinity. The transcripts built from Cufflinks and Stringtie were filtered using the same approach previously ${ }^{27,59}$. To compare alternative splicing in W22 and B73, we took the transcripts from the $\mathrm{B} 73 \mathrm{v} 4$ annotation that includes alternative splicing isoforms, and mapped back to the W22v2 genomes and called the alternative splicing loci in $\mathrm{B} 73$ relative to the $\mathrm{W} 22 \mathrm{v} 2$ genome.

Mapping of $\boldsymbol{M} \boldsymbol{u}$ and $\boldsymbol{D} \boldsymbol{s}$ insertion sites. Germinal $M u$ insertions from 13,444 UniformMu lines ${ }^{7,9}$ were mapped in the B73v4 and $\mathrm{W} 22 \mathrm{v} 2$ genomes using the Illumina-based MuSeq protocol as described in an earlier study ${ }^{8}$. Insertion sites that were assigned unique locations in the W22 genome were analyzed to determine the presence and locations of corresponding sites in the B73v4 genome. Correspondence of insertion sites in W22 and B73 was based on shared MuSeq reads. Insertion sites in W22 identified by MuSeq reads that failed to align to B73 were scored as W22-specific. Flanking Ds (fDs) sequences, which derive from sequenced clones, were placed on each genome assembly using the processing pipeline and placement quality criteria described previously ${ }^{13}$ with minor modifications. For each genome, the pipeline was first applied to the unmasked genome and unmasked fDs sequences, and then the remaining, unplaced fDs sequences were repeat masked and run through the pipeline again with the unmasked genome. Multiple fDs from the same insertion event were collapsed into a single placement when at least one fDs clone included a sequence directly adjacent to the $D s$ insertion site. Repeat masking used Repeatmasker as described previously ${ }^{13}$

DNA methylation and chromatin accessibility. Whole-genome bisulfite sequencing and analysis. Genomic DNA $(1 \mu \mathrm{g})$ was sheared to a size of $200-300 \mathrm{bp}$. These DNA fragments were then used to construct a whole-genome bisulfite sequencing library using the KAPA library preparation kit (KK8232). Briefly, the DNA fragments were subjected to end repair, A-tailing, adapter ligation and dual-SPRI size selection following the manufacturer's instructions. The resulting library, which has a size between $250 \mathrm{bp}$ and $450 \mathrm{bp}$, was treated with bisulfite sodium so that unmethylated cytosine could be converted to uracil using the Zymo EZ DNA methylation lightning kit (D5031). The KAPA HiFi HotStart Uracil + (KK2801) was used in the PCR reaction with the following program: $95^{\circ} \mathrm{C}$ for $2 \mathrm{~min}, 8 \mathrm{cycles}$ of $98^{\circ} \mathrm{C}$ for $30 \mathrm{~s}, 60^{\circ} \mathrm{C}$ for $30 \mathrm{~s}, 72^{\circ} \mathrm{C}$ for $4 \mathrm{~min}$ and a final extension step at $72^{\circ} \mathrm{C}$ for $10 \mathrm{~min}$. Finally, the PCR-enriched library was cleaned up using SPRI beads. The library was sequenced using Illumina HiSeq2000 with the PE mode and 100 cycles and the reads are available at SRX3136383. Trim_glore was used to trim adapter sequences and read quality was assessed with the default parameters and PE reads mode. Reads that passed quality control were aligned to the W22 assembly using Bismark, allowing at most 1 mismatch in the 25 bp seed sequence (-N 1 -L 25). Duplicate reads were detected and removed using Picard tools. Reads that are uniquely mapped and that are properly paired were used to extract methylation status at each individual cytosine using bismark_methylation_extractor. For PCR inserts that have a size of less than $200 \mathrm{bp}$ (so that the two sequencing reads might overlap), only the first read was used to calculate the methylation level. The file with methylation information for individual cytosines was used to create 100-bp non-overlapping sliding windows across the W22 chromosomes for each of the three sequence contexts, CG, $\mathrm{CHG}$ and $\mathrm{CHH}(\mathrm{H}=\mathrm{A}, \mathrm{C}$ or T). Within each 100-bp window, the total number of methylated and unmethylated reads for every cytosine was summed for each sequence context, and methylation levels were derived using the following formula: (no. methylated_reads/(no. methylated_reads + no. unmethylated_reads))

MNase assays and analysis. Seeds were sown into Fafard 3 soil mix in two $11^{\prime \prime} \times 21$ " $\times 2.5$ " plastic trays. Nine days after planting, above-ground tissue was cut at the soil line and flash frozen in two batches (one per tray) and stored at $-80^{\circ} \mathrm{C}$ until grinding. The two batches of frozen seedlings were ground under liquid nitrogen with a mortar and pestle and crosslinked by stirring for $10 \mathrm{~min}$ in $100 \mathrm{ml}$ ice-cold fixation buffer ( $15 \mathrm{mM}$ Pipes- $\mathrm{NaOH}$ at $\mathrm{pH} 6.8,0.32 \mathrm{M}$ sorbitol, $80 \mathrm{mM} \mathrm{KCl}, 20 \mathrm{mM} \mathrm{NaCl}, 0.5 \mathrm{mM}$ EGTA, $2 \mathrm{mM}$ EDTA, $1 \mathrm{mM}$ dithiothreitol, $0.15 \mathrm{mM}$ spermine and $0.5 \mathrm{mM}$ spermidine) containing $1 \%$ formaldehyde. Fixation was stopped by adding glycine to $125 \mathrm{mM}$. Nuclei were isolated by adding Triton $\mathrm{X}-100$ to $1 \%$ final by the addition of $0.1 \mathrm{vol}$ of a $10 \%$ (vol $/ \mathrm{vol}$ ) Triton X-100 stock, followed by stirring for $10 \mathrm{~min}$. The suspension was filtered through one layer of Miracloth (Calbiochem) and placed in $50 \mathrm{ml}$ centrifuge tubes. In these centrifuge tubes, $35 \mathrm{ml}$ nuclear suspensions were underlaid with a $15 \mathrm{ml}$ Percoll cushion composed of $50 \%$ (vol/vol) Percoll (GE) in BFA. Nuclei suspensions were centrifuged at $3,000 \mathrm{~g}$ for $15 \mathrm{~min}$ at $4{ }^{\circ} \mathrm{C}$. The nuclei at the Percoll interface were transferred to a $50 \mathrm{ml}$ tube and diluted twofold with MNase digestion buffer (50 mM Tris- $\mathrm{HCl}$ at $\mathrm{pH} 7.5,320 \mathrm{mM}$ sucrose, $4 \mathrm{mM} \mathrm{MgCl}$ and $1 \mathrm{mM} \mathrm{CaCl}_{2}$ ). Nuclei suspensions were centrifuged at $2,000 \mathrm{~g}$ for $10 \mathrm{~min}$ at $4{ }^{\circ} \mathrm{C}$, and nuclei pellets were resuspended in $2.5 \mathrm{ml}$ MNase digestion buffer. Nuclei were aliquoted into $500 \mathrm{ml}$ aliquots, flash-frozen in liquid nitrogen and stored at $-80^{\circ} \mathrm{C}$. Nuclei were thawed at room temperature and digested by adding MNase to $10 \mathrm{U} \mathrm{ml}^{-1}$ (light) or $100 \mathrm{U} \mathrm{ml}^{-1}$ (heavy), and incubated at room temperature for $5 \mathrm{~min}$. Digestions were stopped with $10 \mathrm{mM}$ EGTA. Nuclei were de-crosslinked by incubation overnight at $65^{\circ} \mathrm{C}$ in the presence of $1 \%$ SDS and $100 \mu \mathrm{g} \mathrm{ml}^{-1}$ proteinase $\mathrm{K}$. DNA was extracted by phenol-chloroform extraction followed by ethanol precipitation. Digested DNA was resuspended in $40 \mathrm{~g} \mathrm{~m} \mathrm{~m}^{-1}$ RNAse A and size-selected with a two-sided Ampure XP bead purification step $(0.5 \times$ beads:DNA followed by $2.5 \times$ beads:DNA). Size-selected DNA was used to prepare sequencing libraries using the NEBNext Ultra DNA Library Prep Kit for Illumina (NEB), using the manufacturer's instructions. Indexed libraries were pooled and sequenced on four Illumina HiSeq 2500 lanes with PE 50-cycle sequencing. Data are available in NCBI SRA (accession SRP118121).

Metaplots for chromatin surrounding genes or TE insertion sites. For DNA methylation, the context-specific levels of DNA methylation for each 100-bp bin across the genome were utilized. For chromatin accessibility, the normalized (reads per million) values for the heavy and light digest were calculated for each 100-bp bin and the ratio (heavy/light) was used as a measure of relative accessibility. For each annotated attribute (gene, TIR, LTR), bedtools closest was used to calculate the distance of each 100-bp bin to that attribute. Relative distance was then determined for the 100-bp bins within the attribute (normalized to a 1,000-bp window). All 100-bp bin data for $1 \mathrm{~kb}$ upstream and downstream of the attribute were retained and data points were averaged for every 100-bp window; these values were then plotted across the genomic region relative to the gene, TIR or LTR attribute. Metaplots of chromatin features surrounding potential $D s$ or $M u$ insertion sites were performed by selecting the 100-bp bins upstream and downstream $(10 \mathrm{~kb})$ of each insertion site using bedtools. Data points were averaged for each 100-bp window across the genomic space relative to the insertion site (indicated by $x$-axis zero).

Analysis of chromatin at edges of TIR TE families. The DNA methylation and MNase data for 100-bp bins were compared with the TIR annotation gff using bedtools closest to identify the 100-bp bins within $4 \mathrm{~kb}$ of each TIR element. Only 100-bp bins that are closest to a single TIR element are used for analysis of chromatin properties at the edge of TIR elements. The TE family ID was identified and for each family (only those families with $10+$ members were used to generate 
the figure; see Supplementary Table 8 for details on these families), the average level of DNA methylation or chromatin accessibility was calculated for each 100bp window with respect to the TE edge. $M u$ and $D s$ data were then added for each DNA methylation context and MNase. The heatmap was then generated using a Euclidean clustering system focusing on the CG and CHG methylation levels. Once the family order was determined, a separate scale was used to generate each context of methylation and MNase to view the trend within each $(\mathrm{CG} / \mathrm{CHG}=0-1$, $\mathrm{CHH}=0-0.15$, MNase $=0-4)$.

Reporting Summary. Further information on experimental design is available in the Nature Research Reporting Summary linked to this article.

Data availability. The W22v2 genome sequence is available at NCBI under the accession GCA_001644905.2. The W22 annotations are available at MaizeGDB.org. There are also RNA-seq (SRR1986376), whole-genome bisulfite (SRX3136383) and MNase (SRP118121) data sets available at NCBI SRA.

\section{References}

42. Zhang, M. et al. Preparation of megabase-sized DNA from a variety of organisms using the nuclei method for advanced genomics research. Nat. Protoc. 7, 467-478 (2012).

43. Avni, R. et al. Wild emmer genome architecture and diversity elucidate wheat evolution and domestication. Science 357, 93-97 (2017)

44. Campbell, M. S. et al. MAKER-P: a tool kit for the rapid creation, management, and quality control of plant genome annotations. Plant Physiol. 164, 513-524 (2014).

45. Law, M. et al. Automated update, revision, and quality control of the maize genome annotations using MAKER-P improves the B73 RefGen_v3 gene models and identifies new genes. Plant Physiol. 167, 25-39 (2015).

46. Wang, B. et al. Unveiling the complexity of the maize transcriptome by single-molecule long-read sequencing. Nat. Commun. 7, 11708 (2016).
47. Fu, L., Niu, B., Zhu, Z., Wu, S. \& Li, W. CD-HIT: accelerated for clustering the next-generation sequencing data. Bioinformatics 28, 3150-3152 (2012).

48. Martin, J. A. et al. A near complete snapshot of the Zea mays seedling transcriptome revealed from ultra-deep sequencing. Sci. Rep. 4, 4519 (2014)

49. Tello-Ruiz, M. K. et al. Gramene 2016: comparative plant genomics and pathway resources. Nucleic Acids Res. 44, D1133-40 (2016).

50. Stanke, M. et al. AUGUSTUS: ab initio prediction of alternative transcripts. Nucleic Acids Res. 34, W435-W439 (2006).

51. Salamov, A. A. \& Solovyev, V. V. Ab initio gene finding in Drosophila genomic DNA. Genome Res. 10, 516-522 (2000).

52. Wicker, T. et al. A unified classification system for eukaryotic transposable elements. Nat. Rev. Genet. 8, 973-982 (2007).

53. Ellinghaus, D., Kurtz, S. \& Willhoeft, U. LTRharvest, an efficient and flexible software for de novo detection of LTR retrotransposons. BMC Bioinformatics 9, 18 (2008)

54. Han, Y., Burnette, J. M. 3rd \& Wessler, S. R. TARGeT: a web-based pipeline for retrieving and characterizing gene and transposable element families from genomic sequences. Nucleic Acids Res. 37, e78 (2009).

55. Ye, C., Ji, G. \& Liang, C. detectMITE: A novel approach to detect miniature inverted repeat transposable elements in genomes. Sci. Rep. 6, 19688 (2016).

56. Wenke, T. et al. Targeted identification of short interspersed nuclear element families shows their widespread existence and extreme heterogeneity in plant genomes. Plant Cell 23, 3117-3128 (2011).

57. Tang, H. et al. SynFind: compiling syntenic regions across any set of genomes on demand. Genome Biol. Evol. 7, 3286-3298 (2015).

58. Schnable, J. C. Genome evolution in maize: from genomes back to genes. Annu. Rev. Plant Biol. 66, 329-343 (2015).

59. Mei, W., Boatwright, L., Feng, G., Schnable, J. C. \& Barbazuk, W. B. Evolutionarily conserved alternative splicing across monocots. Genetics 207 465-480 (2017). 


\section{Life Sciences Reporting Summary}

Date:

April, 192018

Nature Research wishes to improve the reproducibility of the work we publish. This form is published with all life science papers and is intended to promote consistency and transparency in reporting. All life sciences submissions use this form; while some list items might not apply to an individual manuscript, all fields must be completed for clarity.

For further information on the points included in this form, see Reporting Life Sciences Research. For further information on Nature Research policies, including our data availability policy, see Authors \& Referees and the Editorial Policy Checklist.

\section{- Experimental design}

\section{Sample size}

Describe how sample size was determined.

For the primary experiments the sample size was 1 (for the genome assembly and annotation). For other experiments the sample size was the population of lines in which novel TE insertions had been characterized.

\section{Data exclusions}

Describe any data exclusions.

No data was excluded

3. Replication

Describe whether the experimental findings were reliably reproduced.

Most experiments were not replicated as they report the results of genome assembly and annotation. Chromatin data was assessed through biological replication and comparisons of profiles at multiple sites.

\section{Randomization}

Describe how samples/organisms/participants were allocated into experimental groups.

\section{Not relevant to this study}

\section{Blinding}

Describe whether the investigators were blinded to group allocation Blinding was not relevant for this study during data collection and/or analysis.

Note: all studies involving animals and/or human research participants must disclose whether blinding and randomization were used.

\section{Statistical parameters}

For all figures and tables that use statistical methods, confirm that the following items are present in relevant figure legends (or the Methods section if additional space is needed).

n/a $\mid$ Confirmed

$\square$ The exact sample size $(n)$ for each experimental group/condition, given as a discrete number and unit of measurement (animals, litters, cultures, etc.) A description of how samples were collected, noting whether measurements were taken from distinct samples or whether the same sample was measured repeatedly.

A statement indicating how many times each experiment was replicated

The statistical test(s) used and whether they are one- or two-sided (note: only common tests should be described solely by name; more complex techniques should be described in the Methods section)

A description of any assumptions or corrections, such as an adjustment for multiple comparisons

$\square$ The test results (e.g. $p$ values) given as exact values whenever possible and with confidence intervals noted

A summary of the descriptive statistics, including central tendency (e.g. median, mean) and variation (e.g. standard deviation, interquartile range) Clearly defined error bars 
Policy information about availability of computer code

\section{Software}

Describe the software used to analyze the data in this study.

The DeNovoMagic2 is propietary software developed by NRGene. A summary of the method is detailed in Online Methods. Other software used in this study is publicly available and described.

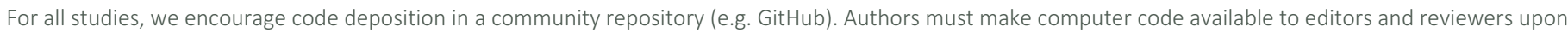
request. The Nature Methods guidance for providing algorithms and software for publication may be useful for any submission.

\section{- Materials and reagents}

Policy information about availability of materials

8. Materials availability

Indicate whether there are restrictions on availability of unique materials or if these materials are only available for distribution by a for-profit company.

\section{Antibodies}

Describe the antibodies used and how they were validated for use in the system under study (i.e. assay and species).

10. Eukaryotic cell lines

a. State the source of each eukaryotic cell line used.

b. Describe the method of cell line authentication used.

c. Report whether the cell lines were tested for mycoplasma contamination.

d. If any of the cell lines used in the paper are listed in the database of commonly misidentified cell lines maintained by ICLAC, provide a scientific rationale for their use.
Seed was sourced from Dr. Hugo Dooner who maintained the stocks and deposited materials at the USDA GRIN under accession PI674445. This stock is freely available at: https://training.ars-grin.gov/gringlobal/ AccessionDetail.aspx?id=1925431

No antibodies were used

No eukaryotic cell lines were used

No eukaryotic cell lines were used

No eukaryotic cell lines were used

No eukaryotic cell lines were used

\section{- Animals and human research participants}

Policy information about studies involving animals; when reporting animal research, follow the ARRIVE guidelines

\section{Description of research animals}

Provide details on animals and/or animal-derived materials used in the study.

Policy information about studies involving human research participants

\section{Description of human research participants}

Describe the covariate-relevant population characteristics of the human research participants.
No animals were used

Research did not involve human subjects 